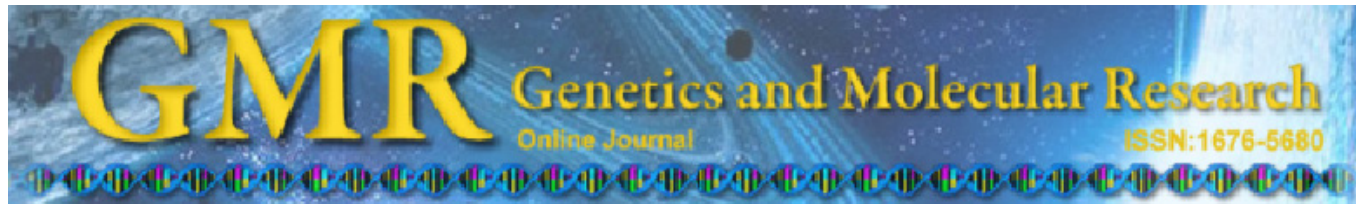

Short Communication

\title{
Plasmid construction for genetic modification of dicotyledonous plants with a glycolate oxidizing pathway
}

\author{
X.L. Bai, D. Wang, L.J. Wei and Y. Wang \\ College of Life Science, Nankai University, Tianjin, China \\ Corresponding author: Y. Wang \\ E-mail: ywanglab222@gmail.com
}

Genet. Mol. Res. 10 (3): 1356-1363 (2011)

Received January 13, 2011

Accepted April 20, 2011

Published July 6, 2011

DOI 10.4238/vol10-3gmr1262

\begin{abstract}
There are many kinds of dicotyledonous $\mathrm{C}_{3}$ plants, which often release $\mathrm{CO}_{2}$ fixed by photosynthesis and consume energy in photorespiration. In Escherichia coli, glycolate can be metabolized by an oxidation pathway that has some of the same compounds as dicotyledonous photorespiration. With the bacterial glycolate metabolism pathway, photorespiration of dicotyledonous plants is genetically modified for less $\mathrm{CO}_{2}$ release and more biomass. In this study, two plasmids involved in this modification were constructed for targeting two enzymes of the glycolate oxidizing pathway, glyoxylate carboligase and tartronic semialdehyde reductase, and glycolate dehydrogenase in Arabidopsis thaliana mitochondria in this pathway. All three enzymes are located in chloroplast by transit peptide derived from Pisum sativum small unit of Rubisco. So far, some crops have been transformed by the two plasmids. Through transformation of the two plasmids, photosynthesis of dicotyledonous plants may be promoted more easily and release less $\mathrm{CO}_{2}$ into the atmosphere.
\end{abstract}

Key words: Plasmid construction; Dicotyledonous plants; Genetic modification; Glycolate oxidizing pathway 


\section{INTRODUCTION}

Many vegetables, fruits, economic crops, and urban greening plants are dicotyledons. The majority of them are $\mathrm{C}_{3}$ plants with loss of energy and release of $\mathrm{CO}_{2}$ during photorespiration, such as cabbage, tea, cotton, apple, soybean, and poplar. In $\mathrm{C}_{3}$ plants, $\mathrm{O}_{2}$ and $\mathrm{CO}_{2}$ compete for the same active site of the difunctional enzyme ribulose-1,5-bisphosphate carboxylase/oxygenase (Rubisco), which can catalyse both the carboxylation and oxygenation of ribulose-1,5-bisphosphate (RuBP) (Bowes et al., 1971). Phosphoglycerate (PGA), the product of RuBP carboxylation by Rubisco, is processed into carbohydrate in photosynthesis. However, photorespiration begins with the oxygenation of RuBP by Rubisco to form phosphoglycolate (PG) as well as PGA. PGA can enter the Calvin cycle directly, whereas PG enters the photorespiration cycle. Plants can recycle part of the carbon by photorespiration, in which PG is metabolized to glycerate, which can re-enter the Calvin cycle (Leegood et al., 1995).

Photorespiration is important for carbon recycling from PG (Keys, 1986; Leegood et al., 1995) and energy dissipation in order to prevent photoinhibition (Kozaki and Takeba, 1996), but it is a wasteful metabolic pathway in general. Oxygenation of RuBP is accompanied by consumption of ATP and reducing equivalents in the form of $\mathrm{NAD} /(\mathrm{P}) \mathrm{H}^{+}$(Maroco et al., 2000). Ammonia formed in mitochondria has to be refixed with extra-ATP in chloroplast. Furthermore, $25 \%$ of the carbon from PG is lost as $\mathrm{CO}_{2}$ in mitochondria through the photorespiration pathway (Leegood et al., 1995).

The photorespiration pathway of $\mathrm{C}_{3}$ plants proceeding in the chloroplast, peroxisome and mitochondria is represented in Figure 1. Many bacteria can metabolize glycolate, the primary product of the oxygenase activity of Rubisco in plant chloroplast, as the sole carbon source. Escherichia coli is one of the bacteria that has the glycolate oxidizing pathway (Lord, 1972; Pellicer et al., 1996). The first step is oxidization of glycolate to glyoxylate catalyzed by glycolate oxidase $(\mathrm{GO})$. And the following reaction catalyzed by glyoxylate carboligase (GCL) condenses two molecules of glyoxylate to tartronic semialdehyde and releases $\mathrm{CO}_{2}$. Tartronic semialdehyde is reduced to glycerate by tartronic semialdehyde reductase (TSR) in E. coli.

In a previous study, the E. coli glycolate oxidizing pathway was introduced into $\mathrm{Ara}$ bidopsis thaliana chloroplast by transformation of GO, GCL and TSR for greater biomass and reduced release of $\mathrm{CO}_{2}$ (Kebeish et al., 2007). Additionally, photosynthesis was enhanced with an increased chloroplastic $\mathrm{CO}_{2}$ concentration in the vicinity of Rubisco while photorespiration was reduced. This was a complex task because bacterial GO is constituted from three different protein subunits, which are encoded by three different genes known as $g l c D, g l c E$ and $g l c F$, all of which are located in the glc operon of E. coli (Pellicer et al., 1996). In fact, they transform 5 independent expression cassettes in all containing protein coding sequences in plant chloroplast.

We replaced the GO with glycolate dehydrogenase (GDH) from A thaliana mitochondria. GDH encoded by the $g d h$ gene can catalyze the same reaction as GO, and it can complement any subunit of GO in E. coli (Bari et al., 2004). Furthermore, gdh encodes a single protein instead of several subunits. These features of $g d h$ simplify the genetic modification of dicotyledonous plants with $g c l$ and $t s r$ from $E$. coli and $g d h$ from A. thaliana. We can modify dicotyledonous plants by transformation of two vectors rather than three. 


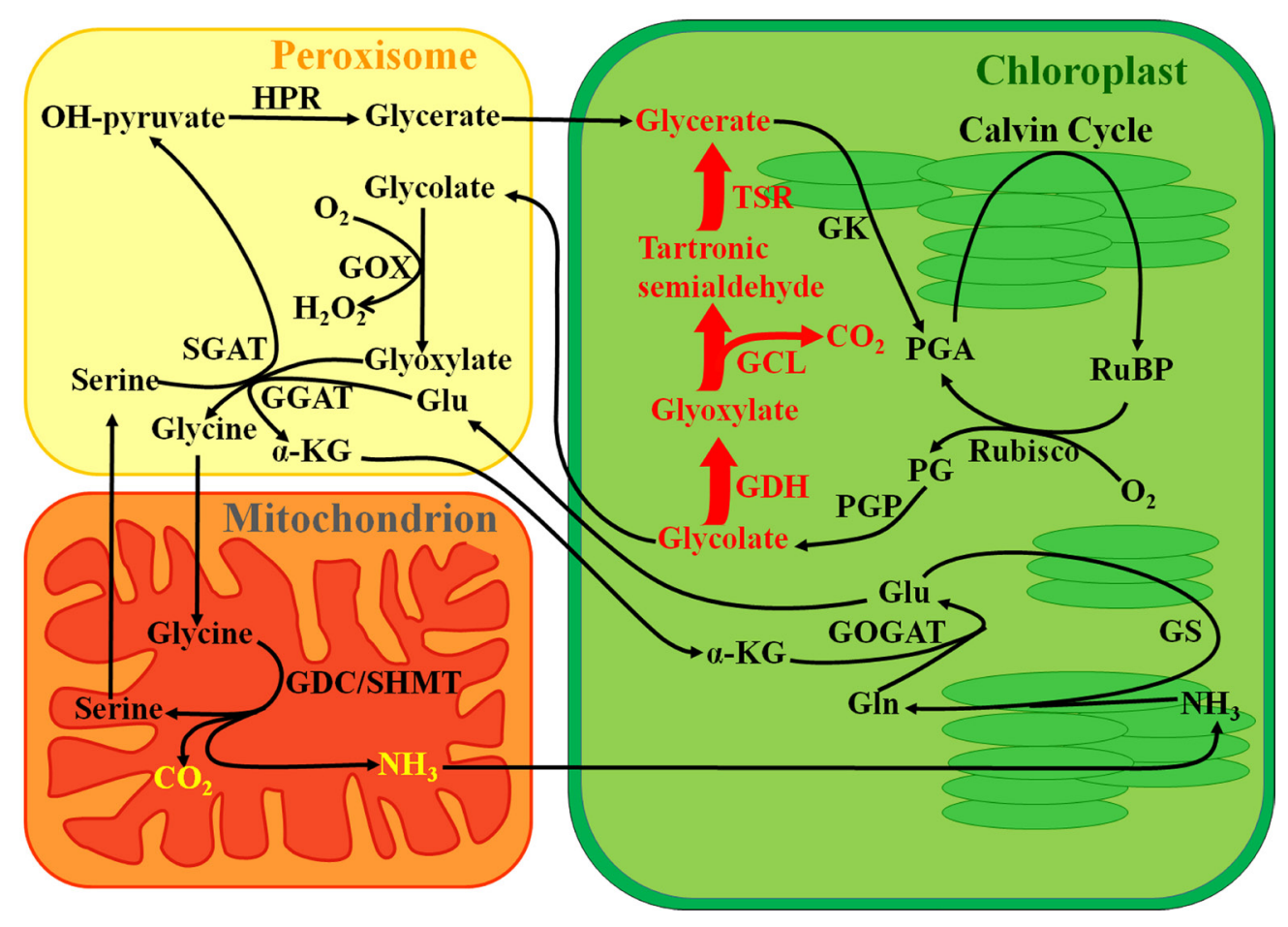

Figure 1. Schematic integration of photorespiration (black) and Escherichia coli glycolate metabolic pathway (red). Rubisco $=$ ribulose-1,5-bisphosphate carboxylase/oxygenase; RuBP $=$ ribulose-1,5-bisphosphate; PGP = phosphogycolate phosphatase; $\mathrm{PG}=$ phosphoglycolate; $\mathrm{PGA}=$ phosphoglycerate $; \mathrm{GOX}=$ glycolate oxidase; GGAT = glyoxylate/glutamate aminotransferase; $\alpha-\mathrm{KG}=$ ketoglutarate; Glu = glutamate; Gln = glutamine; GDC/ SHMT $=$ glycine decarboxylase/serine hydroxymethyl transferase $;$ SGAT $=$ serine/glyoxylate aminotransferase; $\mathrm{HPR}=$ hydroxypyruvate reductase; $\mathrm{OH}$-pyruvate = hydroxypyruvate; GK = glycerate kinase; GS = glutamine synthetase; GOGAT = glutamate/oxoglutarate aminotransferase; GDH = glycolate dehydrogenase; GCL = glyoxylate carboxyligase; TSR = tartronic semialdehyde reductase.

Firstly, a universal expression vector pUE was constructed, which was modified from the commercial vector pBlueScript SK+ (pSK). pUE contains the coding sequence of dicotyledonous chloroplast transit peptide (CTP) for targeting a protein into chloroplast, between the duplicated cauliflower mosaic virus $(C a M V) 35 S$ promoter $(2 \times 35 \mathrm{~S})$ and nopaline synthase gene (nos) polyA (PA) terminator sequences. The $c t p$ sequence was cloned from the Pisum sativum small unit of Rubisco $(\mathrm{RbcS})$ while $35 S$ promoter and nos polyA sequences were cloned from binary vector pCAMBIA 1301 . Secondly, the $g d h, g c l$ and $t s r$ sequences were inserted into pUE multiple cloning sites (MCS) between $c t p$ and nos polyA, respectively, resulting in three individual expression vectors. Thirdly, $g c l$ and $t s r$ expression cassettes were inserted into one vector pCAMBIA 1301 tandemly, yielding a binary expression vector p1301DiGT. Meanwhile, the $g d h$ expression cassette was inserted into another vector pPGN, yielding another binary expression vector pPGNG. The backbones of pCAMBIA 1301 and pPGN offer hygromycin and kanamycin resistance to transgenic plants. Genetic modification of dicotyledonous plants will be established by co-transformation of the two final binary expression vectors to receptor plants. 


\section{MATERIAL AND METHODS}

\section{Strains and vectors}

E. coli DH5 $\alpha$ was used as the recipient strain for recombinant plasmids. pMD ${ }^{\circledR}$ 19-T Simple Vector (TaKaRa) is a commercial T vector without MCS. pSK is a common cloning vector, which offers the backbone of the universal expression vector pUE. Both pCAMBIA 1301 and pPGN are binary expression vectors and show different antibiotic resistance in plants.

\section{DNA and RNA manipulation}

RNA was extracted from plants by TRIZOL regent (TIANGEN). Restriction enzymes were used as the conditions recommended by protocols (TaKaRa). Klenow fragment for blunting 3' ends of DNA and alkaline phosphatase for dephosphorylation of DNA were also bought from TaKaRa.

The DNA fragments were isolated with the TIANgel Midi Purification Kit according to manufacturer instructions (TIANGEN). The plasmid DNA or ligation mixtures were transformed into DH5 $\alpha$ competent cells (TIANGEN). All DNA fragments were sequenced by Sangon and alignments were done to identify its correctness by Nucleotide BLAST Tool in NCBI.

\section{Polymerase chain reaction (PCR)}

The primers used in this study for PCR amplification are shown in Table 1, synthesized by Sangon. PCR was performed in $20 \mu \mathrm{L}$ mixtures as follows: plasmid DNA (1 10 ng), 1X PCR buffer, $0.2 \mathrm{mM}$ of each dNTP, $200 \mathrm{nM}$ of each primer and $0.25 \mu \mathrm{L}$ TransTaq DNA Polymerase High Fidelity (TRANS). The PCR program was carried out as follows: initial denaturation at $95^{\circ} \mathrm{C}$ for $5 \mathrm{~min}$, followed by $30 \sim 35$ cycles of $95^{\circ} \mathrm{C}$ for $30 \mathrm{~s}$, appreciate annealing temperature for $30 \mathrm{~s}$, and $72^{\circ} \mathrm{C}$ for $2 \mathrm{~min}$, and $72^{\circ} \mathrm{C}$ for $10 \mathrm{~min}$ after the cycles.

\section{RESULTS}

\section{Clone of involved elements and genes}

We amplified the full-length of the $g d h$ coding sequence from $A$. thaliana transcriptome, $g c l$ and $t s r$ sequences from the $E$. coli genome. Both $2 \times 35 \mathrm{~S}$ promoter and nos PA were cloned from the pCAMBIA 1301 vector. The $c t p$ fragment was amplified from the $P$. sativum genome. The sequences were all inserted into the $\mathrm{T}$ vector with designed restriction endonuclease site as shown in Table 1. Every cloned fragment, including regulatory elements and genes, was validated by sequencing.

These $\mathrm{T}$ vectors harboring involved elements and genes are named by prefixing ' $\mathrm{T}$ ' to their names, as in T- $g c l$, T-PA, T-35S, and T-ctp. 
X.L. Bai et al.

1360

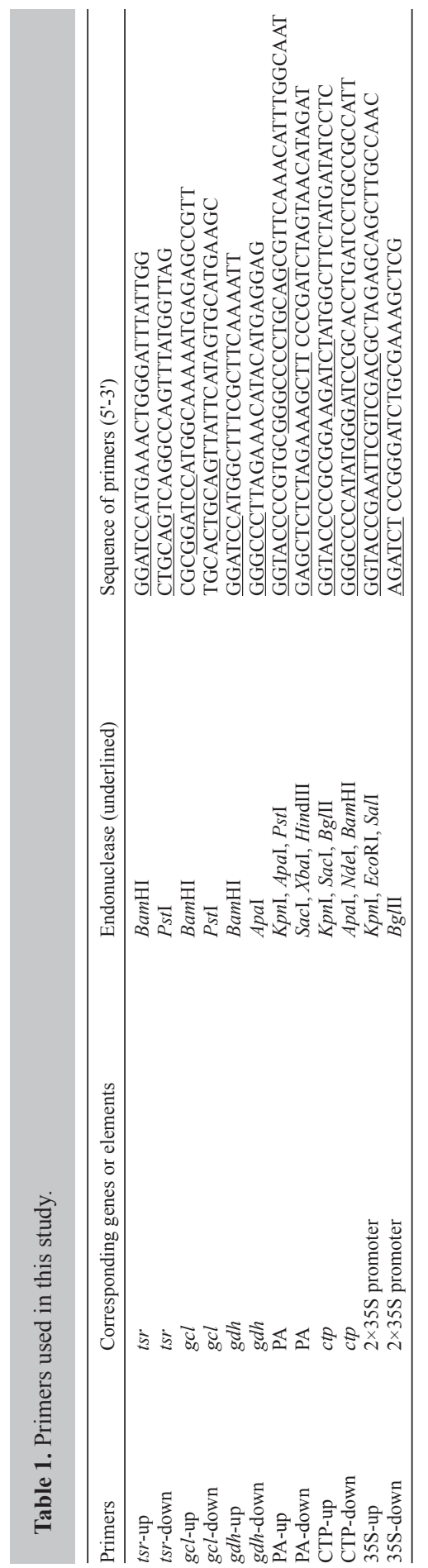

Genetics and Molecular Research 10 (3): 1356-1363 (2011)

CFUNPEC-RP www.funpecrp.com.br 


\section{Construction of universal expression vector pUE}

We constructed a universal vector pUE, which can target protein under control of its promoter and terminator into dicotyledonous plant chloroplast.

pSK is the backbone of pUE, so involved elements and genes were added to it. The PA fragment digested from T-PA with KpnI and SacI was cloned to pSK MCS, which was then digested with the same enzymes, yielding pSK-PA. The ctp and 35S fragments from T-ctp and T-35S, digested with $K p n \mathrm{I} / A p a \mathrm{I}$ and $K p n \mathrm{I} / B g l \mathrm{II}$, respectively, were tandemly inserted into pSK-PA by the same method, yielding pUE (Figure 2A).
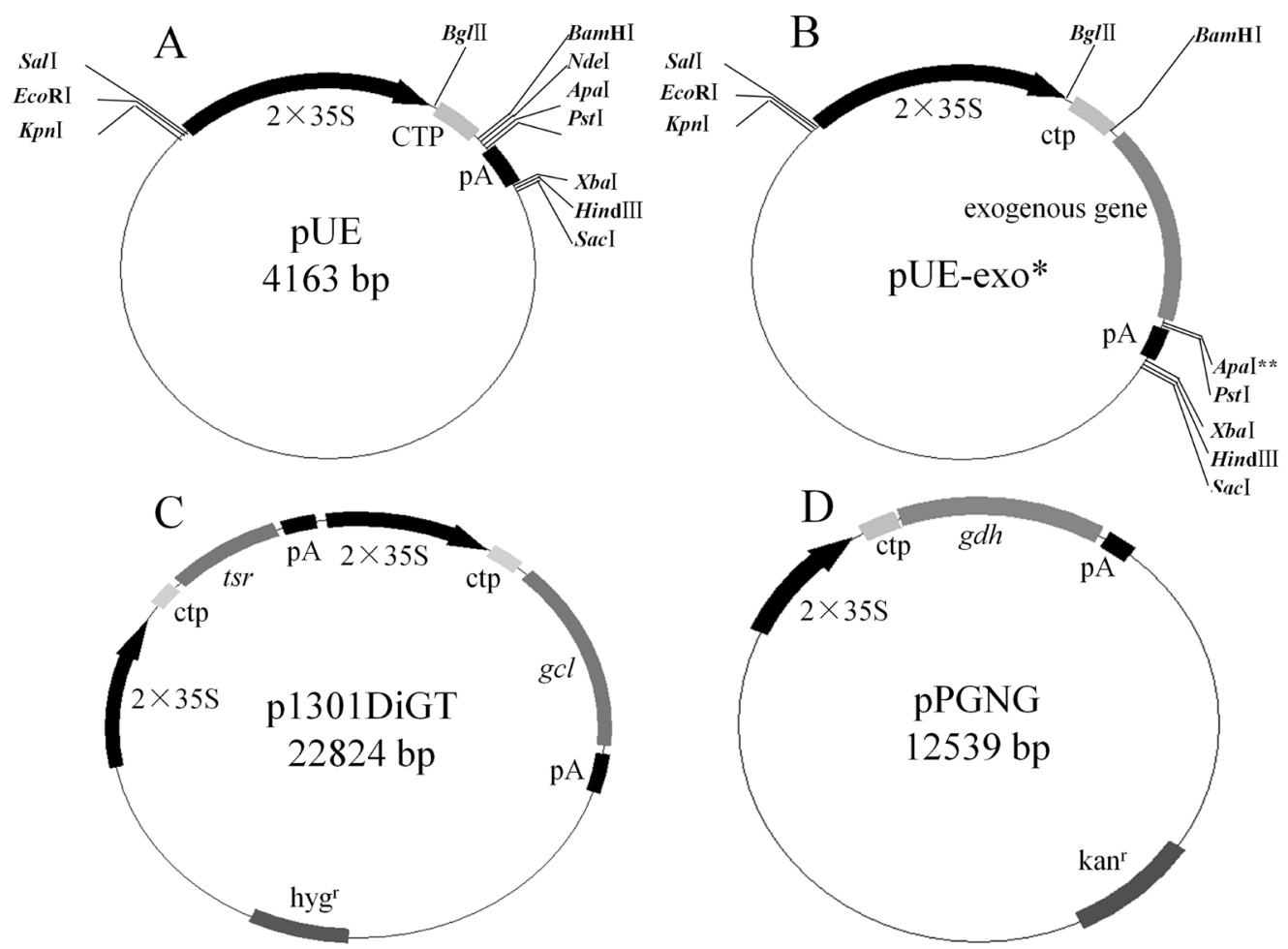

Figure 2. The structure of the primary vectors in this study. A. Universal expression vector pUE. B. pUE containing exogenous gene in MCS. C. Binary expression vector p1301DiGT. D. Binary expression vector pPGNG. *Exo represents the three exogenous genes involved in this study. ${ }^{* *}$ This endonuclease site only exists in pUE-gdh.

\section{Construction of cassettes of exogenous genes}

pUE has an MCS between $c t p$ and PA. All the exogenous genes, $g c l$, tsr and $g d h$, were inserted into MCS of pUE, with digestion of restriction enzymes designed in the primer sequences of genes involved (Table 1) from corresponding T vectors, to form their expression cassettes (Figure 2B). The vectors containing expression cassettes of exogenous genes were named by adding the suffix "pUE" to the gene name, with their pUE origination and backbone indicated. 


\section{Construction of dicotyledonous binary expression vectors}

pCAMBIA 1301 is a common commercial binary expression vector, offering hygromycin resistance when expressed in plants and an MCS for DNA insertion. The order of enzyme sites in this MCS is EcoRI-SacI-KpnI-BamHI-XbaI-SalI-PstI-HindIII.

pUE-tsr was digested by $X b a$ I and self-ligated following 3' end blunting and dephosphorylation. The $t s r$ expression cassette fragment from pUE-tsr was ligated into the MCS of pCAMBIA 1301 by digestion of HindIII and SalI, and then the $g c l$ expression cassette fragment originating from $\mathrm{pUE}-\mathrm{gcl}$ was ligated to the formed vector MCS by digestion of EcoRI and $\mathrm{XbaI}$, yielding p1301DiGT (Figure 2C).

pPGN is another binary expression vector, offering hygromycin resistance to plants and a KpnI-SmaI-BamHI-XbaI MCS. The $g d h$ expression cassette from pUE-gdh was ligated to pPGN MCS by digestion of KpnI and XbaI, yielding pPGNG (Figure 2D).

pPGNG and p1301DiGT were examined by PCR and restriction enzyme digestion to make sure their sequences were correct (Figure 3).

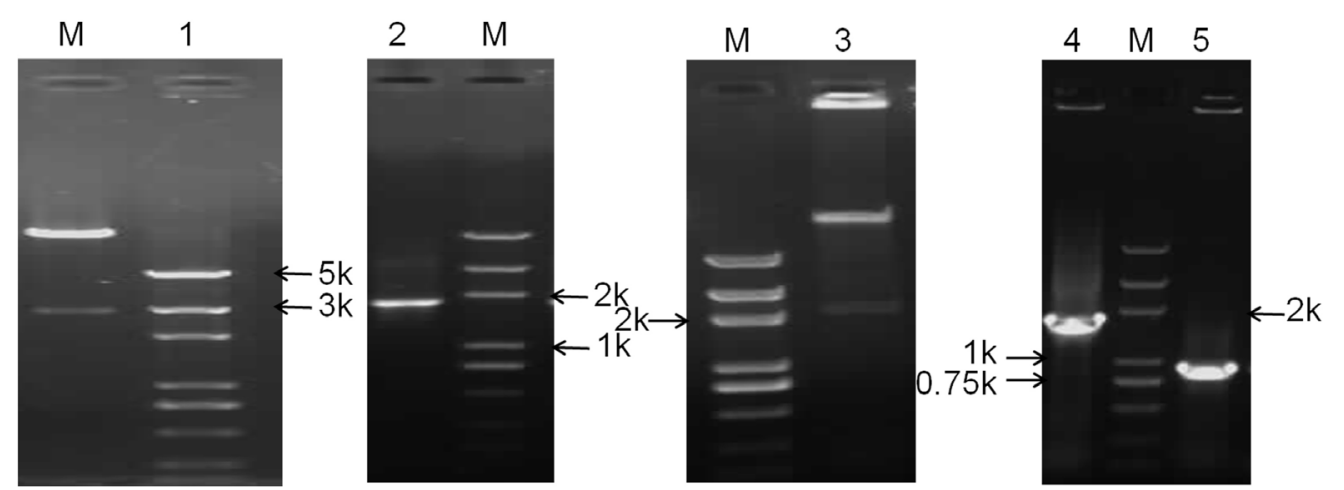

Figure 3. Tests of PCR assay and restriction endonuclease analysis of pPGNG and p1301DiGT. Lane $M=$ DNA Marker 2Kplus (TRANS); lane $1=$ digestion of pPGNG with KpnI and XbaI; lane 2 = PCR with primers $c t p$-up and $g d h$-dn using pPGNG as template; lane $3=$ digestion of p1301DiGT with PstI; lane $4=$ PCR with primers $g c l$-up and $g c l$-dn using p1301DiGT as template; lane $5=$ PCR with primers $t s r$-up and $t s r$-dn using p1301DiGT as template.

\section{DISCUSSION}

Previous study was done for genetic modification of dicotyledonous plants with a glycolate oxidizing pathway, which may improve dicotyledonous plant photosynthesis and photorespiration for greater biomass and lower $\mathrm{CO}_{2}$ release. Our strategy of replacing $\mathrm{GO}$ with GDH and transforming only two vectors instead of three is much easier. Agrobacterium tumefaciens strains, which contain p1301DiGT and pPGNG, were obtained for further application on vegetables, fruits, economic crops, and urban greening plants. Besides economic benefits, application of this strategy may also reduce carbon release into the atmosphere, which is a severe ecological problem for humans. 


\section{REFERENCES}

Bari R, Kebeish R, Kalamajka R, Rademacher T, et al. (2004). A glycolate dehydrogenase in the mitochondria of Arabidopsis thaliana. J. Exp. Bot. 55: 623-630.

Bowes G, Ogren WL and Hageman RH (1971). Phosphoglycolate production catalysed by ribulose diphosphate carboxylase. Biochem. Biophys. Res. Commun. 45: 716-722.

Kebeish R, Niessen M, Thiruveedhi K, Bari R, et al. (2007). Chloroplastic photorespiratory bypass increases photosynthesis and biomass production in Arabidopsis thaliana. Nat. Biotechnol. 25: 593-599.

Keys AJ (1986). Rubisco: its role in photorespiration. Phil. Trans. R. Soc. Lond. 313: 325-336.

Kozaki A and Takeba G (1996). Photorespiration protects C3 plants from photooxidation. Nature 384: 557-560.

Leegood RC, Lea PJ, Adcock MD and Häusler RE (1995). The regulation and control of photorespiration. J. Exp. Bot. 46: 1397-1414.

Lord JM (1972). Glycolate oxidoreductase in Escherichia coli. Biochim. Biophys. Acta 267: 227-237.

Maroco JP, Ku MSB and Edwards GE (2000). Utilization of $\mathrm{O}_{2}$ in the metabolic optimization of $\mathrm{C}_{4}$ photosynthesis. Plant Cell Environ. 23: 115-121.

Pellicer MT, Badia J, Aguilar J and Baldoma L (1996). glc locus of Escherichia coli: characterization of genes encoding the subunits of glycolate oxidase and the glc regulator protein. J. Bacteriol. 178: 2051-2059. 